\title{
Study of Relative Exposure to Natural Disaster Risk
}

\author{
Chongfu Huang ${ }^{1,2,3}$ \\ ${ }^{1}$ Key Laboratory of Environmental Change and Natural Disaster, Ministry of Education, Beijing Normal University, Beijing 100875, China \\ ${ }^{2}$ State Key Laboratory of Earth Surface Processes and Resources Ecology (Beijing Normal University), Beijing 100875, China \\ ${ }^{3}$ Academy of Disaster Reduction and Emergency Management, Faculty of Geographical Science, Beijing Normal University, Beijing \\ 100875, China \\ hcongfu@bnu.edu.cn
}

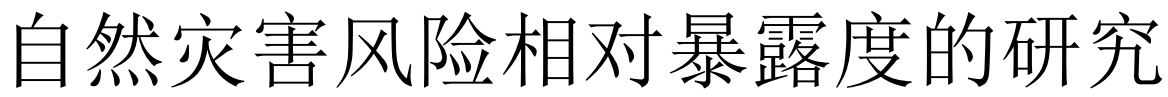

\author{
黄崇福 $1,2,3$ \\ ${ }^{1}$ 北京师范大学, 环境演变与自然灾害教育部重点实验室, 北京 100875 , 中国 \\ 2 北京师范大学, 地表过程与资源生态国家重点实验室, 北京 100875, 中国 \\ 3 北京师范大学, 地理科学学部, 减灾与应急管理研究院, 北京 100875 , 中国 \\ hcongfu@bnu.edu.cn
}

\begin{abstract}
In view of the need of relative exposure, as background data, in the geospatial information diffusion model, this paper defines "exposure to natural disaster risk" and "relative exposure". Dividing "the product of the disaster and the parameter affecting the disaster with respect to a risk bearing body lightly affected by disaster" by "the product to a body severely affected", we quantify the exposure of the body lightly affected relative to the body severely affected. The study shows that risk exposure to natural disasters consists of three parts: the exposure to risk source, the exposure to vulnerability, and the exposure to value. Statistical data can be used to make a scale for measuring the relative exposure of each geographic unit in the risk zone.
\end{abstract}

Keywords-information diffusion, risk bearing body, relative exposure, risk source, disaster, background data

摘要一针对地理空间信息扩散模型中相对暴露度背景数据 的需要，本文定义了 “自然灾害风险暴露” 和 “相对暴 露”。灾情较低者的灾情与影响灾情的参数之积, 除以灾情 较重者的相应之积, 得灾情较低者相对灾情较重者的暴露 度。研究表明, 自然灾害风险暴露, 包括三个部分：对风险 源的暴露、脆弱性暴露和价值暴露。用统计资料制作的标 尺, 能衡量风险区各地理单元的相对暴露度。

关键词一信息扩散，风险承受体，相对暴露，风险源，灾 情, 背景数据

\section{I. 引言}

用地理空间信息扩散模型[1]推测空白地理单元上的 灾情，有助于解决信息孤岛问题，是大灾中精准救助的 关键技术之一。由于该模型具有非线性识别能力, 且能 学习矛盾样本, 较之加权地理回归[2]和人工神经元网络 [3], 能更适于在复杂的灾害系统中用样本学习出较客观 的因果关系。

地理空间信息扩散模型, 是将灾区已观测的地理单 元上的背景数据和灾情形成的样本, 视为小样本, 用正 态信息扩散公式[4]，对其进行集值化处理，构造出 “背 景数据” 和 “灾情”之间的因果关系，从而帮助人们， 用空白地理单元上的背景数据, 推导出灾情。
以背景数据为桥梁, 将已观测数据携带的信息, 扩 散到空白单元的地理空间信息扩散模型, 能否发挥作 用, 与所用背景数据, 密切相关。背景数据是地理特征 在理单元上的属性值, 但只有依据其可合理估计空白地 理单元上某待估值时, 这些属性值, 才能称为此待估值 的背景数据。换言之, 只有背景数据和待估值具有明显 的因果关系时, 背景数据才有意义, 背景数据才能发挥 桥梁作用。

当地理空间信息扩散模型用于推测空白单元上的灾 情时，“人口”、“人均 GDP” 和 “相对暴露度” 是最 关键的三个背景数据。然而, 如何获得 “相对暴露度” 这一背景数据, 目前并无一定之规。

本文从推测灾情的需要出发, 讨论什么是 “相对暴 露度”, 并探讨如何衡量此背景数据, 用于地理空间信 息扩散模型。

\section{II. 风险暴露与相对暴露度}

“暴露” (Exposure) 是一个使用频率很高的词语, 最 生动的使用，莫过于军事用语 “暴露了目标”。人员、 装备、工事等, 一旦暴露, 就使自己成为敌人的靶子。 乔装改扮, 隐蔽得很好, 就没有暴露目标。“暴露” 的 基本词义是指露在外面, 无所遮蔽。

“风险暴露” (Risk Exposure) 则是一个专业词汇, 存 在诸多释意。

现代风险分析的标志事件是 1970 年美国庆祝了第一 个地球日，美国政府同时设立了环境保护署，全面关注 环境风险问题。在环境 (空气、水、土地和其它自然资 源）风险评价中, “暴露” 指的是可能被伤害的对象与 有害化学品的接触。

核风险暴露, 由当量、距离和持续时间决定。核武 器的冲击和爆炸, 数公里到数十公里内是致命的全覆 盖, 暴露度由核武当量决定; 辐射暴露与距离平方成反 比, 呈指数减小 [5]; 辐射影响暴露, 与被辐射的持续时 间有关。 
在职业健康风险评价中, 又有外暴露和内暴露之 分。外暴露评价主要是计算有毒物质泄漏后浓度场分布 [6]; 内暴露评价主要是计算各组织或器官中化学物及代 谢物浓度随时间的变化, 尤其是模拟肝脏等组织或器官的 代谢转化过程, 以提供化学物在体内的分布和转化数据 [7]。

在金融系统中，虽然特定风险的暴露不一样，例 如, 信用风险的暴露是指受到信用风险影响的贷款量, 而利率或汇率风险的暴露则是受到利率或汇率变动影响 的资产和负债的价值，但金融风险的 “暴露” 可概括为 在各种业务活动中容易受到风险因素影响的资产和负债 的价值 [8], 或说暴露在风险中的头寸状况。此 “风险暴 露”也被形象地译为 “风险敞口”。

在自然灾害风险分析领域, “暴露”的概念比较混 乱，例如，文献[9]直接将 “承灾体” 称为 “暴露性”, 而 2013 年发布的一个 “全球暴露数据库” 中将 “建筑 物、居住或在其中工作的人口” 明确为 “暴露资产” [10], 而不是简单地称这些资产为 “暴露” 。研究洪水 灾害的文献[11]认为, 暴露是在可能发生灾害的地方存 在人类及其生计、资产、基础设施和资源。国内有学者 干脆建议不要使用 “暴露”这一术语, 而用 “孕灾环 境” 取而代之。目前, 灾害领域大多数研究人员眼中的 “暴露”，其实类似于金融领域的 “敞口”。

与职业健康风险分析中人体对于危化品的 “暴露” 不同，自然灾害风险分析中风险承受体对于灾害（地 震、洪水等）的 “暴露” 因其风险源的不确定性很大, 灾害风险暴露不可能由风险承受体来度量。

承灾体是指承受灾害的对象, 而风险承受体是指承 受风险的对象。承灾体须承受灾害, 但风险承受体可能 承灾, 也可能不承灾。承受弱小风险源, 或风险源较 远, 或足够结实的风险承受体, 不会承灾。例如, 核爆 影响范围内的人员是承灾体, 但外围人员只是风险承受 体。核爆之前的这两类人员，均是风险承受体。

严格意义上讲, 任何风险暴露都由风险源和风险承 受体两者来决定。面对复杂的灾害系统, “暴露”不可 能简化为 “风险承受体”。

在将自然灾害和其风险分别定义为 “由自然事件或 力量为主因造成的生命伤亡和人类社会财产损失的事 件” 和 “由自然事件或力量为主因导致的末来不利事件 情景” [4]的基础上, 本文给出自然灾害风险暴露的定义 如下:

定义 1. 自然灾害风险暴露是生命和人类社会财产 在未来是否容易遭受自然事件或力量打击的一种状态。

例如, 一个村子的地震风险暴露, 是指村民及财产 是否容易在未来的地震出现震害的一种状态。远离震中 或震级不高, 当然不会出现震害; 房子足够结实, 也不 容易出现震害。在这两种情况下, 该村子的地震风险暴 露度都很小, 甚至可以忽略不计。根据定义 1 可知, 一 个村子的地震风险暴露度, 并不由村子的人口和财富来 决定。

灾害风险暴露, 类似于在伏击中, 我们不能说 “暴 露” 是曾经隐蔽的 “土兵”, 两者不能划等号, 只能说 “土兵暴露” 了, 暴露只是士兵的一种状态, 这种状态
与是否存在敌人有关。没有敌人, 无所谓暴露与否。将 “生命和人类社会财产” 视为 “士兵”，将 “自然事件 或力量” 视为 “敌人”, 理解定义 1 就很容易了。

显然, 度量 “敞口” 容易, 度量 “暴露” 并不容 易。只有在锁定或假设了风险源的前提下，才可用 “风 险承受体” 来度量 “暴露”。一旦风险源的确切位置、 暴发时间和强度为已知, 相应的风险, 就转化为 “伪风 险” ，“能被打击到的对象” 才能直接视为 “灾害风险 暴露”。如果仅仅只知道哪些对象是风险承受体, 但其 面对的风险源位置不确定, 或暴发时间不确定, 或暴发 强度不确定, 则度量 “暴露”, 十分困难。

退而求其次, 度量 “相对暴露”, 会比度量 “暴 露”容易一些。

定义 2. 设 $A, B$ 是两个面对同一风险源的风险承受 体。如果此风险源暴发时, $A$ 比 $B$ 更容易遭受打击, 称 $A$ 的相对暴露比 $B$ 高。

例如, 对易发洪水的某一河流而言, 离河较近的村 子, 相对暴露较高; 地势较高的村子, 相对暴露较低。 约定相对暴露度最高的村子其相对暴露度为 1 , 其它村 子的相对暴露度, 就可以通过与最高者的比较而确定下 来。

只有两个风险承受体与同一风险源的关系完全相同 时, 他们自身的属性, 才会左右各自的相对暴露度。在 同一设防水平下, 人口和财富较多的风险承受体的相对 暴露度比人口和财富较少者高。

\section{III. 由风险承受体与风险源关系决定的相对暴露度}

设 $A 、 B$ 两个风险承受体面对的风险源是 $O$ 。在 $A$ 、 $B$ 的内在属性完全相同时, 我们研究如何根据它们与风 险源的关系, 来确定相对暴露度。这里的内在属性, 是 指地理位置和环境以外的, 诸如设防水平、人口、经济 和社会、地质、地形和地貌等的所有属性。

距离, 是风险承受体与风险源最重要关系。不同灾 种的距离概念是不同的。对地震风险源而言, 距离是指 风险承受体到震中的距离, 简称震中距; 对河水泛滥成 灾的洪水风险源而言, 距离是指风险承受体到最近河堤 的距离; 对台风风险而言, 距离是指风险承受体与台风 最近路径中心的距离。

除距离外, 风险源到风险承受体的路径是很重要的 关系。与震中位于同一地震断裂带上的风险承受体, 更 容易遭受破坏; 同样的距离情况下, 经常决堤附近的村 庄, 更容易遭受破坏; 台风所过之处, 两边的灾情, 时 常会有不同。

其次, 风险承受体周围的环境, 有时也会左右灾 情。周围有活动断裂带的风险承受体, 震害会加重; 泛 洪区中的村庄, 比其它村庄, 更容易遭受破坏; 背坡的 风险承受体，常能躲过台风的破坏。

不失一般性, 设 $\alpha_{1}, \alpha_{2}, \ldots, \alpha_{n}$ 是表达风险承受体 与风险源 $n$ 个关系的相应参数。例如, 可用 $\alpha_{1}$ 来表达风 险承受体与风险源之间的距离; 可用 $\alpha_{2}$ 来表达从风险源 到风险承受体的路径其打击力的衰减系数; 可用 $\alpha_{3}$ 来表 达风险承受体周围的环境对灾情的放大系数, 等等。 
首先, 我们来研究针对上述的一个参数如何确定相 对暴露度。

设 $A 、 B$ 与风险源的关系参数分别是 $\alpha_{A}$ 和 $\alpha_{B}$ 。假定 $A 、 B$ 的灾情只受此参数的影响, 并设为 $y_{A}$ 和 $y_{B}$, 且 $y_{A} \geq y_{B}$ 。

在这种情况下, 根据定义 2 知, $A$ 的相对暴露比 $B$ 高。如果我们将最高暴露度定义为 1 , 则是规定, $A$ 相 对 $B$ 的暴露度为 1 。而 $B$ 相对 $A$ 的暴露度, 应该小于 1 , 具体数值则由归一化原则来确定。

设 $x_{1}, x_{2}, \ldots, x_{t}$ 是 $t$ 个观测值, 归一化它们的最简 单原则是取大归一化:

$$
x_{i}^{\prime}=\frac{x_{i}}{c}, \quad i=1,2, \cdots, t
$$

式中, $c=\max \left\{x_{1}, x_{2}, \ldots, x_{t}\right\}$ 。

灾情 $y$ 的取大归一化值是:

$$
y_{A}^{\prime}=\frac{y_{A}}{y_{A}}=1, \quad y_{B}^{\prime}=\frac{y_{B}}{y_{A}}
$$

当 $\alpha_{A} \geq \alpha_{B}$ 时, 灾情 $y$ 与参数 $\alpha$ 是正相关关系; 反之, 是负相关关系。例如, 震害 $y$ 与周围活动断裂带条数是 正相关关系，而与震中距是负相关关系。 值是:

当灾情 $y$ 与参数 $\alpha$ 是正相关时, 参数 $\alpha$ 的取大归一化

$$
\alpha_{A}^{\prime}=\frac{\alpha_{A}}{\alpha_{A}}=1, \quad \alpha_{B}^{\prime}=\frac{\alpha_{B}}{\alpha_{A}} \leq 1
$$

当灾情 $y$ 与参数 $\alpha$ 是负相关时, 先要确定一个最大相 关值 $a$, 超越此值后, 灾情 $y$ 衰减为 0 。先用 $a$ 对 $\alpha$ 进行 正相关化处理, 令:

$$
\alpha_{A}^{\prime \prime}=1-\frac{\alpha_{A}}{a}, \quad \alpha_{B}^{\prime \prime}=1-\frac{\alpha_{B}}{a}
$$

参数 $\alpha^{\prime \prime}$ 的取大归一化值是:

$$
\alpha_{A}^{\prime \prime \prime}=\frac{\alpha_{A}^{\prime \prime}}{\alpha_{A}^{\prime \prime}}=1, \quad \alpha_{B}^{\prime \prime \prime}=\frac{\alpha_{B}^{\prime \prime}}{\alpha_{A}^{\prime \prime}} \leq 1
$$

显然, 无论是 $y_{B}$ 接近于 $y_{A}$, 还是 $\alpha_{B}$ 接近于 $\alpha_{A}, B$ 的 暴露都会接近于 $A$ 。于是, 我们可用 $y_{B}^{\prime}$ 和 $\alpha_{B}^{\prime}$, 或 $y_{B}^{\prime}$ 和 $\alpha_{B}^{\prime \prime \prime}$ 来定义 $B$ 相对 $A$ 的暴露度 $E_{A(\alpha)}(B)$ :

$$
E_{A(\alpha)}(B)= \begin{cases}y_{B}^{\prime} \alpha_{B}^{\prime}, & \text { 如果 } y, \alpha \text { 正相关 } \\ y_{B}^{\prime} \alpha_{B}^{\prime \prime \prime}, & \text { 如果 } y, \alpha \text { 负相关 }\end{cases}
$$

由于

$$
\begin{gathered}
y_{B}^{\prime} \alpha_{B}^{\prime}=\frac{y_{B}}{y_{A}} \times \frac{\alpha_{B}}{\alpha_{A}}=\frac{y_{B} \alpha_{B}}{y_{A} \alpha_{A}} \\
y_{B}^{\prime} \alpha_{B}^{\prime \prime \prime}=\frac{y_{B}}{y_{A}} \times \frac{\alpha_{B}^{\prime \prime}}{\alpha_{A}^{\prime \prime}}=\frac{y_{B}}{y_{A}} \times \frac{1-\frac{\alpha_{B}}{a}}{1-\frac{\alpha_{A}}{a}}=\frac{y_{B}\left(a-\alpha_{B}\right)}{y_{A}\left(a-\alpha_{A}\right)}
\end{gathered}
$$

于是

$$
E_{A(\alpha)}(B)= \begin{cases}\frac{y_{B} \alpha_{B}}{y_{A} \alpha_{A}}, & \text { 如果 } y, \alpha \text { 正相关 } \\ \frac{y_{B}\left(a-\alpha_{B}\right)}{y_{A}\left(a-\alpha_{A}\right)}, & \text { 如果 } y, \alpha \text { 负相关 }\end{cases}
$$

例如, 设 $A 、 B$ 是两栋完全相同的建筑物。在一次 地震中, 它们的震中距分别是 $\alpha_{A}=10$ 公里和 $\alpha_{B}=50$ 公 里, 它们的震害指数分别是 $y_{A}=0.9$ 和 $y_{B}=0.2$ 。假定该地 震的地震波衰减 80 公里后, 不会再对该类建筑物造成破 坏, 即, 式(6)中的 $a=80$.

显然, 在此例中, 灾情 (震害指数) $y$ 与参数 (震 中距） $\alpha$ 是负相关关系，即， $\alpha$ 值越大，则 $y$ 值就越小。 于是, 由式(6)知, 建筑物 $B$ 相对建筑物 $A$ 的暴露度是:

$$
E_{A(\text { 震中距 })}(B)=\frac{y_{B}\left(a-\alpha_{B}\right)}{y_{A}\left(a-\alpha_{A}\right)}=\frac{0.2(80-50)}{0.9(80-10)}=0.095
$$

只要我们将灾情最严重的风险承受体的暴露度定义 为 1 , 用式(6)就可以计算出其它多个风险承受体的相对 暴露度。 度。

其次, 我们来研究针对两参数如何来确定相对暴露

设 $\alpha_{1}, \alpha_{2}$ 是表达风险承受体与风险源两个关系的参 数, $A$ 与风险源的第 1 个关系的参数记为 $\alpha_{A 1}$, 第 2 个记 为 $\alpha_{A 2}, B$ 与风险源的两关系参数分别记为 $\alpha_{B 1}$ 和 $\alpha_{B 2}$, 同 样假没 $A 、 B$ 的灾情是 $y_{A} \geq y_{B}$ 。

用式(6), 我们可以由灾情和两个关系参数分别计算 出 $B$ 相对 $A$ 的暴露度, 记为 $E_{A, \alpha_{1}}(B)$ 和 $E_{A, \alpha_{2}}(B)$ 。

通常, 两个关系参数对灾情的影响程度不一样, 我 们可用加权求和计算 $B$ 相 $A$ 的暴露度。设两个关系参数 $\alpha_{1}, \alpha_{2}$ 的权重分别为 $w_{\alpha_{1}}$ 和 $w_{\alpha_{2}}$, 且 $w_{\alpha_{1}}+w_{\alpha_{2}}=1$, 则 $B$ 相对 $A$ 的暴露度是:

$$
E_{A(\alpha)}(B)=w_{\alpha_{1}} E_{A, \alpha_{1}}(B)+w_{\alpha_{2}} E_{A, \alpha_{2}}(B)
$$

对于多个关系参数, 分别对各参数计算出相对暴露 度, 再进行加权求和。

针对关系参数 $\alpha_{i}, i=1,2, \ldots, n$, 设 $B$ 相对 $A$ 的暴露度 分别是 $E_{A, \alpha_{i}}(B), i=1,2, \cdots, n$, 并设这些关系参数对灾情 的影响权重分别是 $w_{\alpha_{i}}, i=1,2, \ldots, n$, 且 $\sum_{i=1}^{n} w_{\alpha_{i}}=1$, 则 $B$ 相对 $A$ 的暴露度是:

$$
E_{A(\alpha)}(B)=\sum_{i=1}^{n} w_{\alpha_{i}} E_{A, \alpha_{i}}(B)
$$

IV. 由风险承受体内在属性决定的暴露度

风险承受体的内在属性, 由两大部分组成, 一是脆 弱性, 二是价值。

建筑物对地震而言, 抗震设防水平和工程地质条件 等, 决定了其脆弱性, 更确切地说, 是易损性。村庄对 洪水而言, 防洪能力、地形和地貌等, 决定了其脆弱 
性。乡镇区域作为风险承受体对河水泛洪而言，平地与 山地的比例，对其脆弱性有显著影响。

虽然生命无价，但仍可计量。例如，风险承受体中 的人员数量，可用来计量生命价值。用货币计量风险承 受体的财产价值, 是一种通行的做法。

由风险承受体内在属性决定的相对暴露, 可细分为 脆弱性相对暴露和价值性相对暴露两大类。

\section{A. 脆弱性相对暴露度}

设风险承受体 $A 、 B$ 与同一风险源的关系完全相 同，但它们对此风险源所属灾种的脆弱性不同，我们研 究如何根据它们的脆弱性, 确定相对暴露度。

设 $\beta_{1}, \beta_{2}, \ldots, \beta_{m}$ 是表达风险承受体脆弱性的 $m$ 个 参数。例如, 对河水泛滥的洪水和沿河乡镇而言, 可 用 $\beta_{1}$ 来表达防洪能力, $\beta_{2}$ 表达地形和地貌是否易成 灾, $\beta_{3}$ 表达平地与山地的比例。

首先, 我们来研究针对一个脆弱性参数如何确定相 对暴露度。

设 $A 、 B$ 的脆弱性参数分别是 $\beta_{A}$ 和 $\beta_{B}$ 。假定 $A 、 B$ 的 灾情只受此参数的影响, 仍设为 $y_{A}$ 和 $y_{B}$, 且 $y_{A} \geq y_{B}$ 。

类似于式(6), 我们推导出 $B$ 相对 $A$ 的脆弱性暴露度 是:

$$
E_{A(\beta)}(B)= \begin{cases}\frac{y_{B} \beta_{B}}{y_{A} \beta_{A}}, & \text { 如果 } y, \beta \text { 正相关 } \\ \frac{y_{B}\left(b-\beta_{B}\right)}{y_{A}\left(b-\beta_{A}\right)}, & \text { 如果 } y, \beta \text { 负相关 }\end{cases}
$$

式中的值 $b$ 表征降低脆弱性的 $\beta$ 的最大有效值。例如, 抗击洪水保护村子的围堰, 并非修得越高越好, 超过一 定的高度，就没有意义。如果以围堰高度作为村子的脆 弱性参数, 它与水灾灾情是负相关, 则 $b$ 为围堰的最大 有效高度。

对脆弱性参数 $\beta_{j}, j=1,2, \ldots, m$, 设 $B$ 相对 $A$ 的暴露度 分别是 $E_{A, \beta_{j}}(B), j=1,2, \cdots, m$, 并设这些脆弱性参数对灾

情的影响权重分别是 $w_{\beta_{j}}, j=1,2, \cdots, m$, 且 $\sum_{j=1}^{m} w_{\beta_{j}}=1$, 同理可知, $B$ 相对 $A$ 的脆弱性暴露度是:

$$
E_{A(\beta)}(B)=\sum_{j=1}^{m} w_{\beta_{j}} E_{A, \beta_{j}}(B)
$$

\section{B. 价值性相对暴露度}

设风险承受体 $A 、 B$ 与同一风险源的关系及脆弱性 完全相同, 但它们的价值不同, 我们研究如何根据它们 的价值, 确定相对暴露度。 度。

我们以财产价值为例, 计算 $B$ 相对 $A$ 的价值暴露

设 $A 、 B$ 的财产价值分别是 $\gamma_{A}$ 和 $\gamma_{B}$ 。假定 $A 、 B$ 的灾 情只受此参数的影响, 仍设为 $y_{A}$ 和 $y_{B}$, 且 $y_{A} \geq y_{B}$ 。
类似于式(6), 并考虑到灾情与风险承受体的财产价 值呈正相关, 我们推导出 $B$ 相对 $A$ 的财产价值暴露度 是:

$$
E_{A(\gamma)}(B)=\frac{y_{B} \gamma_{B}}{y_{A} \gamma_{A}}
$$

由于生命价值与财产价值完全不同，除非保险理赔 的需要, 我们不能用式 (8) 的方法, 加权求和, 计算 $B$ 相对 $A$ 的, 包括生命和财产的价值暴露度。

\section{V. 用统计资料衡量相对暴露度}

上述研究表明, 人们通常使用的, 以风险承受体的 价值表征的暴露, 并非自然灾害风险中的真正暴露。风 险承受体与风险源关系的暴露, 脆弱性表现出来的暴 露, 是风险暴露中的重要成分。价值暴露, 只是暴露的 成分之一。

虽然上述公式须在已知灾情的情况下才能计算出相 对暴露度, 但表达了相对暴露度的物理意义, 也充分说 明, 面对同一风险源, 决定灾情的是暴露度, 而不仅仅 是风险承受体的价值。

将地理空间信息扩散模型用于推测空白地理单元上 的灾情时, 地理单元扮演了 “风险承受体” 的角色, “人口” 和 “人均 GDP” 这两个背景数据间接表达了价 值性暴露度, 只须加上能间接地表达关系参数相对暴露 度和脆弱性相对暴露度的背景数据, 就能通过样本学 习, 构建出背景数据与灾情的因果关系。

显然, 如果能采集到所有的关系参数 $\alpha_{1}, \alpha_{2}, \ldots, \alpha_{n}$ 和脆弱性参数 $\beta_{1}, \beta_{2}, \ldots, \beta_{m}$, 则不需具体计算各地理 单元的相对暴露度, 直接作为背景数据使用即可。

现实情况是, 能采集到的关系参数和脆弱性参数很 少, 我们只能针对重要参数, 标定相对暴露度。最简单 的方法，是用统计资料衡量相对暴露度

不失一般性, 设参数 $x$ 对灾情 $y$ 是一个重要的参 数。假定在某次灾害事件后, 人们对同一类的 $N$ 个承灾 体, 记录了它们的此参数值和灾情值, 形成了一个容量 为 $N$ 的样本 $S$ :

$$
S=\left\{\left(x_{1}, y_{1}\right),\left(x_{2}, y_{2}\right), \cdots,\left(x_{N}, y_{N}\right)\right\}
$$

假定用某种统计模型处理此样本, 我们获得了一个 输入-输出关系:

$$
y=f(x)
$$

对同一灾种的某风险区, 假设从 $M$ 个地理单元 $g_{1}$, $g_{2}, \ldots, g_{M}$ 对某风险源 $O$ 采集得参数 $x$ 的数值分别是:

$$
z_{1}, z_{2}, \cdots, z_{M}
$$

并且我们假定样本 $S$ 的输入值和风险区的参数值已 经从小到大排序，即，

$$
x_{1} \leq x_{2} \leq \cdots \leq x_{N} ; \quad z_{1} \leq z_{2} \leq \cdots \leq z_{M}
$$

当 $f$ 正相关时, 意味着, 在历史事件中, 具有参数 $x_{N}$ 的承灾体, 相对暴露度最大; 而在风险区中, $z_{M}$ 所 在地理单元的暴露度最大。 
当我们将各地理单元中最大暴露度定义为 1 时, 如 果视 $f$ 为一杆 “称”, 并标定灾情为 $f\left(z_{M}\right)$ 时, 暴露度 是 1 。于是, 风险区中地理单元的相对暴露度分别是:

$$
\begin{aligned}
& E_{g_{M}(x)}\left(g_{1}\right)=\frac{f\left(z_{1}\right)}{f\left(z_{M}\right)}, \\
& E_{g_{M}(x)}\left(g_{2}\right)=\frac{f\left(z_{2}\right)}{f\left(z_{M}\right)}, \\
& \cdots, \\
& E_{g_{M}(x)}\left(g_{M-1}\right)=\frac{f\left(z_{M-1}\right)}{f\left(z_{M}\right)} \\
& E_{g_{M}(x)}\left(g_{M}\right)=1
\end{aligned}
$$

当 $f$ 负相关时, 意味着, 在历史事件中, 具有参数 $x_{1}$ 的承灾体, 相对暴露度最大; 而在风险区中, $z_{1}$ 所在 地理单元的暴露度最大。此时, 由统计关系 $f$ 衡量出的 相对暴露度分别是:

$$
\begin{aligned}
& E_{g_{1}(x)}\left(g_{1}\right)=1, \\
& E_{g_{1}(x)}\left(g_{2}\right)=\frac{f\left(z_{2}\right)}{f\left(z_{1}\right)}, \\
& \cdots, \\
& E_{g_{1}(x)}\left(g_{M}\right)=\frac{f\left(z_{M}\right)}{f\left(z_{1}\right)}
\end{aligned}
$$

\section{VI. 结论与讨论}

自然灾害风险暴露是生命和人类社会财产在未来是否 容易遭受自然事件或力量打击的一种状态。灾害发生 后，容易度量 “承灾体” 的 “暴露”。灾害发生前，只 能度量 “相对暴露”。两个面对同一风险源的风险承受 体，更容易遭受打击者的相对暴露度较高。

风险承受体与风险源关系的暴露、脆弱性表现出来 的暴露和价值暴露，共同组成了风险暴露。一个因素影 响暴露度的程度, 等同于该因素影响灾情的程度。

灾情较低者的灾情与影响灾情的参数之积, 除以灾 情较重者的相应之积, 得灾情较低者相对灾情较重者的 暴露度。

厘清了相对暴露度的物理意义后, 我们可以用统计 资料来衡量风险区各地理单元的相对暴露度, 为地理空 间信息扩散模型推测空白单元上的灾情, 提供背景数据 中的 “相对暴露度”。

随着现代科技的发展, 自然灾害风险分析和风险管 理的研究, 进入了 “基于数据, 超越经验” 的时代, 同 时, 也出现了 “忽视实体, 热衰虚化” 的现象。

事实上，灾害风险分析领域的研究，大抵可分为三 他层次:

第一层: 风险源，风险承受体，...
第二层：脆弱性，暴露， $\ldots$

第三层：综合风险，韧性，...

如果没有第一层次中对相关问题研究出来的实体数 据, 对第二层次中相关问题的研究, 只能泛于空谈, 甚 至概念混乱。

如果没有第一层次中的实体数据和第二层次中的丰 富研究成果, 第三层次中的研究, 只能是概念炒作及形 形色经验的展示。

虚化的研究类似于虚拟经济, 会热闹一时, 但基础 的研究类似于实体经济, 才是提高科技竞争力的根本。 作为一个承前启后的研究环节, 风险暴露的研究, 尚有 许多未明之处, 但愿本文的研究, 能给读者提供有益的 参考。

\section{致谢}

本项目受国家重点研发计划课题 “重大自然灾害多 层级精准救助关键技术研究”（编号： 2017YFC1502902）和国家自然科学基金项目 “独立多灾 种年度财产损失综合风险的信息扩散合成范式研究”

（编号：41671502）联合资助。

\section{参考文献}

[1] 黄崇福, “借助背景数据的地理空间信息扩散技术,” J. Risk Anal. Crisis Resp., vol. 9, issue 1, pp.2-10, 2019.

[2] D. J. Lieske, and D. J. Bender, "A robust test of spatial predictive models: geographic cross-validation," J. Environ. Inf., vol. 17, issue 2, pp. 91-101, 2011.

[3] B. Purkait, S. S. Kadam, and S. K. Das, "Application of artificial neural network model to study arsenic contamination in groundwater of Malda District, Eastern India," J. Environ. Inf., vol.12, issue 2, pp.140-149, 2008.

[4] 黄崇福, 自然灾害风险分析与管理. 北京: 科学出版社, 2012 年, pp. 220-221.

[5] M. P. Atkinson, Z. Cao, and L. M. Wein, "Optimal stopping analysis of a radiation detection system to protect cities from a nuclear terrorist attack," Risk Analysis, vol. 28, issue 2, pp. 353-371, 2008.

[6] 李敏嫣, 黄德寅, 刘茂, “基于 MCSim 软件的苯职业暴露健康风险 分析研究,” J. Risk Anal. Crisis Resp., vol. 3, issue 3, pp. 135-145, 2013.

[7] 张倩, 黄德寅, 刘茂, 李敏嫣, “基于暴露相关剂量估算模型的内暴 露模拟研究,” J. Risk Anal. Crisis Resp. , vol. 3, issue 4, pp. 175-184, 2013.

[8] Basel Committee, International Convergence of Capital Measurement and Capital Standards: A Revised Framework. Basel, Switzerland: Bank for International Settlements, 2005.

[9] 张璐, 锡林郭勒盟草原干旱灾害风险综合分析. 呼和浩特: 内蒙古师范大学, 2015.

[10] A. D. Bono, and M.G. Mora, "A global exposure model for disaster risk assessment," Int. J. Disaster Risk Reduction, vol. 10, pp. 442-451, 2014.

[11] Y. Qiang, "Flood exposure of critical infrastructures in the United States,” Int. J. Disaster Risk Reduction, vol. 39, 101240, 2019. 